\title{
Changes to the packing structure of type I collagen from non-enzymatic glycation.
}

\section{Rama S. Madhurapantula, Joseph Orgel \\ Illinois Institute of Technology}

Homeostasis of cells in tissues is maintained through interactions with the constituent extracellular matrix (ECM) components, at least in part. Changes to these ECM structures and in particular to collagen play an pivotal role in the progression of various pathologies, and make them potential targets for therapeutic intervention. However, information on specific changes to these ECM structures is sparse.

Non-enzymatic glycosylation, also known as glycation, results in the formation of sugar-mediated covalent crosslink called Advanced Glycation Endproducts (AGEs). The chemistry of these products has been identified decades ago, but their structure and location within the D-periodic packing structure of collagen remains unknown. Glycation occurs in all mammals but is accelerated in diabetics which leads to secondary pathologies. Modified boronic acid compounds have been used historically to determine the concentrations of AGEs in globular and fibrillar proteins using fluorescent chromatographic methods.

As in most seminal contributions that elucidated the molecular and ultrastructural features of collagens, we utilized X-ray diffraction and the process of Multiple Isomorphous Replacement (MIR) and molecular replacement to using the m-dansylamino phenylboronic acid (mDPBA) to elaborate on the location of AGEs. Analyzing these data is often intricate and has been previously reported for other collagens using antibodies as MIR derivatives. Similar analyses were performed in the current study to detect glycation mediate crosslinks to type I collagen in rat tail tendons. We also present the possible downstream effects of these crosslinks on binding of ligands, cell interaction and associated pathologies, for instance impaired wound healing in diabetics. 